\title{
Isolation and characterization of mouse embryonic stem cell line by parthenogenesis
}

\author{
Shumin $\mathrm{Yu}^{1,2}$, Xinrong Yan $^{1}$, Dongmei Chen ${ }^{1}$, Huayan Wang ${ }^{1}$, Zhongying Dou ${ }^{1}$ \\ ${ }^{1}$ Shaanxi Branch of National Stem Cell Engineering \& Technology Center, Northwest A\&F University, Yangling, Shaanxi 712100, \\ China; ${ }^{2}$ Animal Medical College, Sichuan Agricultural University, Yaan, Sichuan 625000, China
}

Parthenogenetic embryos are an alternative resource to establish the autologous embryonic stem cell (ES) lines. Additionally, parthenogenesis oriented ES cells can be used to study pluripotency of ES cells, gene regulation and imprinting of $\mathrm{X}$ chromosome. We used the female Kunming mice mating with male 129/ter mice to generate F1 hybrid female mice. Matophase II oocytes from F1 mice were activated by ethanol and developed to blastocysts in vitro when incubated in GI/GII media for 4 days. To isolate ES cells, parthenogenetic embryos were cocultured with feeder layer of the inactivated mouse embryonic fibroblasts (MEF) until attaching to the dish and forming the undifferentiated ICM outgrowths after 5-7 days. ES cell-like clonies can be observed when continued to co-culture with MEF feeder for 5-7 days after the ICM outgrowths were trypsinized in combination with manual dissection. Our results showed that among 31 blastocysts derived from parthenogenesis, there were 15 embryos that attached to the dish and formed 11 ICM outgrowths with the undifferentiated morphology. From those ICM colonies, we isolated two embryonic stem cell lines. One ES-like cell line, that named P132 and had been carefully stored in liquid nitrogen, was continually cultured up to $40^{\text {th }}$ passages without losing the features of stemness. Moreover, karyotype analysis revealed that the karyotype of P132 was XX and the normal karyotype rate at the passages of $15^{\text {th }}$ and $28^{\text {th }}$ were $85 \%$ and $65 \%$, respectively. The P132 has the alkaline phosphatase activity. The immunohistochemistry assays showed that P132 expressed ES cell marker of SSEA-1, but no SSEA-3 and SSEA-4. P132 also expressed the ES cell specific genes such as nanog, oct4 and gdf-3 when tested by RTPCR. ES-like P132 can form embryoid bodies after 4-6 days in the suspension culture when LIF and feeder cells were removed. When continue to grow P132 after it formed embryoid body, P132 can differentiate into neuronlike, cardiamyocyte-like, fibroblast-like and epitheliallike cells. The immunohistochemistry assays showed that differentiated cells expressed the markers of three germ layers: ectoderm: Nestin, $\beta$-Tubulin III and GFAP; mesoderm: $\alpha$-Actin; endoderm: $\alpha$-AFP. We also used RT-PCR to analysis the expression of several genes in differentiated cells, including fgf-5 and nf-68 for ectoderm, brachyury $T$ for mesoderm and $\alpha$-afp for endoderm. Above results suggested that the isolated P132 cells can differentiate into all types of cells derived from three germ layers. To test the stemness of P132 cells, we injected P132 into nude mice. Within the six weeks after subcutaneous injection, a teratoma with $0.5 \mathrm{~cm}$ diameter was grown at the injection site. The teratoma was analyzed by the histochemistry, and the result showed that the teratoma contained the tissue structure of neuron-like, vascular-like and muscle-like under the observation of microscope. These experimental results indicated that the established ES-like cell line fitted the whole criteria of mouse ES cell. In summary, we established successfully an embryonic stem cell line derived from parthenogenetic embryos of F1 hybrid mouse of $129 / \operatorname{ter} \times \mathrm{KM}$, and the cell line preserved the pluripotency.

Keywords: embryonic stem cells, parthenogenesis, mouse, pluripotency

Cell Research (2008) 18:s120. doi: 10.1038/cr.2008.210; published online 4 August 2008

Correspondence: Zhongying Dou

E-mail: douzhongying@chinaren.com 